\title{
New insights into the proteins interacting with the promoters of silkworm fibroin genes
}

\section{Yan Ma}

Southwest University

Qin Luo

Southwest University

Yao Ou

Southwest University

Yiyun Tang

Southwest University

Wenhui Zeng

Southwest University

Haomiao Wang

Southwest University

Jie Hu

Southwest University

Hanfu Xu ( $D$ xuhf@swu.edu.cn )

Southwest University

\section{Research Article}

Keywords: Silkworm, Fibroin gene, Promoter, DNA-interacting protein, Regulation

Posted Date: April 12th, 2021

DOl: https://doi.org/10.21203/rs.3.rs-400247/v1

License: (c) (i) This work is licensed under a Creative Commons Attribution 4.0 International License. Read Full License

Version of Record: A version of this preprint was published at Scientific Reports on August 5th, 2021. See the published version at https://doi.org/10.1038/s41598-021-95400-0. 


\section{Abstract}

The silkworm, Bombyx mori, is a silk-producing insect that has contributed greatly to human society. The silk gland of $B$. mori is a specialized organ responsible for synthesizing silk fibroin and sericin proteins under control of numerous factors. However, which factors are involved in direct silk protein synthesis regulation remains largely unknown. We report the identification of promoter-interacting proteins (PIPs) necessary for the direct regulation of genes encoding fibroin proteins, including the fibroin heavy chain $(f i b H)$, fibroin light chain ( $f i b L)$, and a 25-kD polypeptide protein $(P 25)$. In the fourth larval molting stage (M4) or day 5 fifth-instar larvae (L5D5), a total of 198, 292, and 247 or 330, 305, and 460 proteins binding to $f i b H$, fibL and $P 25$, respectively, were identified from the posterior silk gland by DNA pull-down combined with mass spectrometry. Many PIPs were particularly involved in ribosome-, metabolism- and disease-related pathways. Additionally, 135 and 212 proteins were identified as common PIPs of fibH, fibL and P25 in M4 and L5D5, respectively. Among all PIPs, we identified 31 potential transcription factors, such as Y-box and poly A-binding proteins, which play roles in nucleotide binding, ATP binding, or protein folding. This study provides the first in-depth profile of proteins interacting with fibroin gene promoters and contributes to a better understanding of silk protein synthesis regulation.

\section{Introduction}

The temporal and spatial control of gene expression, which is mainly achieved through the precise regulation of gene promoters, is indispensable for many biological processes ${ }^{1}$. The regulation of gene promoters is a rather complex process due to the involvement of various regulatory factors that interact directly with promoters and activate/inhibit their activities. For each target gene, regulatory factors are ubiquitous or tissue-specific proteins that associate with promoter DNA and regulate gene expression through a variety of mechanisms, leading to alternative cell fates and behaviors and controlling organ development ${ }^{2-4}$. Therefore, elucidating the regulatory factors that bind directly to the promoter regions of target genes is a key step toward ascertaining their functions as well as regulatory mechanisms.

The silkworm, Bombyx mori, is a lepidopteran insect of great importance because of its use in silk production and its research value for understanding the biology of insects ${ }^{5}$. The silk gland (SG) of $B$. mori is a specialized organ in which silk proteins are efficiently synthesized and spun out to build cocoons. Generally, the SG is divided into three morphologically and functionally distinct parts: the anterior silk gland (ASG), the middle silk gland (MSG), and the posterior silk gland (PSG) ${ }^{6}$. PSG is the only part that synthesizes fibroin protein, a major component that accounts for approximately $75 \%$ of silk fibers ${ }^{7}$. The regulation of fibroin protein synthesis is particularly interesting and rather complex. In brief, the expression of genes encoding fibroin proteins, including the fibroin heavy chain (fibH), fibroin light chain (fibL), and a 25-kD polypeptide protein $(P 25)$, is synchronously switched on during intermolt larval stages and switched off during molt stages. Remarkably, massive amounts of fibroin protein are rapidly synthesized in PSG cells beginning on the 3 rd day of the fifth instar in larvae ${ }^{8,9}$. Previous studies have suggested that fibroin gene expression is regulated by many regulatory factors at the transcriptional level in a concerted 
manner ${ }^{10,11}$. It is therefore of profound interest to elucidate the factors involved to better understand the regulatory mechanisms of both SG development and silk protein synthesis.

In recent years, great efforts have been made to identify the factors regulating fibroin genes. In particular, transcription factors (TFs) have attracted the most attention due to their vital roles in the transcriptional regulation of gene expression ${ }^{12}$. In B. mori, only a few TFs, including FMBP-1, SGF-2, Bmdimm, POU-M2, and BmFTZ-F1 ${ }^{13-19}$, have been shown to regulate the expression of fibroin genes via direct binding to their promoter regions. A comprehensive understanding of the regulatory factors determining fibroin gene expression is still lacking, especially for those factors that directly interact with fibroin genes.

In this study, we aimed to elucidate the promoter-interacting proteins (PIPs) that regulate fibroin gene transcription and protein synthesis. By using the DNA pull-down strategy combined with mass spectrometry, we identified a large number of PIPs of $\mathrm{fibH}$, fibL, and P25 from the PSG in two representative developmental stages, the fourth larval molting stage (M4) and day 5 fifth-instar larvae (L5D5). Further analyses revealed common and unique PIPs between M4 and L5D5 as well as their functional characteristics and interaction relationships. Overall, these results provide preliminary information and new insights for understanding the regulatory factors associated with fibroin genes.

\section{Results}

Enrichment of PIPs using 5'-biotinylated DNA probes. To identify the PIPs of each fibroin gene in M4 and L5D5 PSGs (Fig. 1a), we first examined the promoter activities of $\mathrm{fibH}$, fibL, and P25 in cultured BmE cells. Significantly, the luciferase activities driven by the promoters of $f i b H$, fibL, and $P 25$ were approximately 133-, 13-, and 20-fold higher than that of the negative control, demonstrating that the promoter region of each fibroin gene contains regulatory sequences related to its expression (Fig. 1b, 1c). Furthermore, we generated 5'-biotinylated DNA probes based on sequences of the fibH, fibL, and $P 25$ promoters shown in Fig. 1c as well as two control probes (Supplementary Fig. S1), and DNA pull-down assays were subsequently performed. As shown in Fig. 1d, multiple proteins from either the M4 or L5D5 PSG showed obvious binding to each of the biotin-labeled DNA probes specific for the $f i b H$, fibL, and $P 25$ promoters in comparison with the control group results, demonstrating that the pull-down assays were effective and successful.

Summary of PIPs determined by HPLC-MS. HPLC-MS was employed to determine the candidate PIPs of each fibroin gene, which are summarized in Table 1 (detailed see Supplementary Table S1). After removing the proteins captured by both negative control DNA probes, we finally identified 198 and 330 PIPs of $\mathrm{fibH}, 292$ and 305 PIPs of $\mathrm{fibL}$, and 247 and 460 PIPs of $P 25$ from the M4 and L5D 5 PSGs, respectively. Notably, the PIPs identified from the L5D5 PSG were much more abundant than those from the M4 PSG, implying that more proteins are recruited to directly regulate the expression of fibroin genes in L5D5, a key period of the efficient synthesis of massive amounts of fibroin protein in the PSG.

Moreover, the number of P25 PIPs identified from the L5D5 PSG was much greater than the numbers of fibH and fibL PIPs, which was a surprising finding and deserves further study. Preliminary Gene Ontology 
(GO) and Kyoto Encyclopedia of Genes and Genomes (KEGG) pathway annotation revealed that the specific PIPs of each group showed some degree of similarity in terms of functional classification (Supplementary Figs. S2 and 3), suggesting that these PIPs may play important roles in the cooperative regulation of fibroin gene expression. Next, we will focus on these common, interesting PIPs to explore their similarities and differences.

Table 1

Summary of candidate PIPs identified by HPLC-MS

\begin{tabular}{|llllll|}
\hline $\begin{array}{l}\text { Gene } \\
\text { promoters }\end{array}$ & $\begin{array}{l}\text { Developmental } \\
\text { stages }\end{array}$ & $\begin{array}{l}\text { Control } \\
\text { group } \\
\text { (GFP } \\
\text { probe) }\end{array}$ & $\begin{array}{l}\text { Control group } \\
\text { (unbiotinylated } \\
\text { probe) }\end{array}$ & $\begin{array}{l}\text { Experimental group } \\
\text { (biotinylated } \\
\text { probe) }\end{array}$ & $\begin{array}{l}\text { Specific } \\
\text { PIPs }\end{array}$ \\
\hline fibH & M4 & 34 & 18 & 232 & 198 \\
\hline fibL & L5D5 & 39 & 23 & 373 & 330 \\
\hline P25 & M4 & 34 & 23 & 325 & 292 \\
\hline & L5D5 & 39 & 77 & 387 & 305 \\
\hline & M4 & 34 & 40 & 291 & 247 \\
\hline
\end{tabular}

Comparison of PIPs between the M4 and L5D5 PSGs. To better understand the characteristics of these PIPs, we first analyzed the common and unique PIPs identified in the M4 and L5D5 PSGs corresponding to each fibroin gene promoter. The results showed that the numbers of common $\mathrm{fibH}$, fibL, and P25PIPs in M4 and L5D5 were 109, 156, and 158, respectively (Fig. 2a and Supplementary Table S2). More than $90 \%$ of these PIPs could be annotated using the BlastKOALA web tool of the KEGG database. Intriguingly, a large proportion of the common PIPs were found to be enriched in three primary pathway categories (i.e., metabolism, particularly metabolic pathways; genetic information processing, especially ribosome and protein processing in the endoplasmic reticulum; and human disease-related pathways, such as those related to prion disease, Huntington's disease, and Parkinson's disease) (Fig. 2b).

Among the unique PIPs (Supplementary Table S3), there were some obvious differences between the fibroin gene promoters and between the two developmental stages. For example, in the M4 PSG, PIPs of $\mathrm{fibH}$ and $\mathrm{fibL}$ were enriched in human disease and genetic information processing pathways, especially those related to the ribosome and RNA transport. PIPs of fibL were also abundant in metabolism pathways, particularly metabolic pathways, while PIPs of $P 25$ were abundant only in pathways involved in transcription, translation, folding, sorting and degradation and replication and repair (Fig. 3a). In the L5D5 PSG, the PIPs of the three fibroin genes were most abundant in disease-related pathways, particularly those related to neurodegenerative disease, followed by metabolism-related categories, such as metabolic pathways and the biosynthesis of secondary metabolites. Some PIPs of fibHand fibL were also enriched 
in the ribosome and protein processing in the endoplasmic reticulum categories, but no PIPs of $P 25$ were enriched in these two pathways (Fig. 3b). In addition, there were many more PIPs identified in the L5D5 PSG than in the M4 PSG, which suggests that more proteins are recruited to ensure the efficient transcription of fibroin genes in the L5 stage, leading to the large-scale synthesis of fibroin proteins.

Identification of common PIPs shared by the three fibroin genes. The expression of fibroin genes is regulated mainly at the transcriptional level in a concerted manner ${ }^{10,11}$. To explore the proteins that may be involved in such coregulation, we analyzed the PIPs that interact with the promoters of all three fibroin genes. The results showed that a large number of PIPs (135 in the M4 PSG and 212 in the L5D 5 PSG) were shared by $f i b H$, fibL and $P 25$ (Fig. 4a and Supplementary Table S4). Functional prediction based on the BlastKOALA web tool of the KEGG database revealed that $90.4 \%(122 / 135)$ and $90.1 \%(191 / 212)$ of the common PIPs received some annotation. These PIPs were annotated to all six KEGG systems, especially genetic information processing, human diseases and metabolism, and some of these PIPs were found in both the M4 and L5D4 PSGs. In addition to organismal systems, there were many more common PIPs in the other five KEGG systems in the L5D5 PSG than in the M4 PSG (Fig. 4b). Further analysis revealed the detailed pathway annotations of those common PIPs identified in M4 and L5D5 (Fig. 5a). The most highly enriched system was human diseases related to neurodegeneration pathways, including the multiple disease, Alzheimer's disease, and Parkinson's disease categories, among others. The second most enriched systems were those of common PIPs involved in the ribosome, protein processing in the endoplasmic reticulum, and metabolic pathways (Fig. 5b).

Functional interaction network of the common PIPs shared by the three fibroin genes. To obtain a greater understanding of the possible functional relationships among the common PIPs identified in the M4 and L5D5 PSGs, we further constructed protein-protein interaction (PPI) networks using the online Search Tool for the Retrieval of Interacting Genes/Proteins (STRING). The initial network of the common PIPs in M4 consists of 132 nodes and 254 edges (Fig. 6a). The vast majority of the nodes were related to 35 PIPs and were mainly involved in ribosome (22 PIPs), RNA transport (5 PIPs), and protein processing in the endoplasmic reticulum (4 PIPs) pathways. The second most significant module (6 PIPs) was involved in ribosome biogenesis in the eukaryotic pathway, and the third most significant module (5 PIPs) was involved in the proteasome pathway. The other modules, which consisted of two or three PIPs, were involved in mismatch repair, aminoacyl-tRNA biosynthesis, DNA replication, nucleotide excision repair, spliceosome, and protein processing in endoplasmic reticulum pathways.

In terms of the common PIPs identified in L5D5, 209 nodes and 444 interactions were found in the initial network (Fig. 6b). The vast majority of the nodes were related to 44 PIPs and were mainly involved in ribosome (25 PIPs) and aminoacyl-tRNA biosynthesis (12 PIPs) pathways, followed by protein export (2 PIPs) and mRNA surveillance (2 PIPs) pathways. Two significant modules consisting of 14 and 9 PIPs were involved in the proteasome and protein processing in the endoplasmic reticulum, respectively. Another significant module consisted of 6 PIPs that were annotated as subunits of the coatomer complex, a cytosolic protein complex that binds to dilysine motifs and is essential for the retrograde Golgi-to-ER transport of dilysine-tagged proteins. The other modules, consisting of two, three, or four PIPs, were 
mainly involved in pathways such as protein processing in the endoplasmic reticulum, protein export, and ribosome biogenesis in eukaryotes. Taken together, these results indicate that the common PIPs identified in M4 and L5D5 PSGs show many interactions among themselves and are at least partially biologically connected as a group. Further studies could lead to a better understanding of their roles in the direct regulation of fibroin gene expression during larval molting and feeding stages.

Description of TFs among the PIPs of fibroin genes. TFs are indispensable for the regulation of fibroin gene transcription and protein synthesis ${ }^{12-19}$. However, only a few TFs from the PSG have been isolated and validated using in vivo/ vitro methods thus far. Therefore, we further identified the TFs among the PIPs of fibroin genes. As summarized in Table 2, 31 potential TFs were identified, which were distributed on 18 chromosomes. The functions of most of these TFs in regulating fibroin gene expression have not been reported. Notably, 5 TFs that could interact with all fibroin gene promoters were identified in both M4 and L5D5, while the numbers of TFs that could interact with all fibroin gene promoters in either M4 or L5D5 were 11 and 10, respectively. Five TFs were found only in M4, while 9 TFs were found only in L5D5. Surprisingly, 20,23, and 29 TFs could interact with the promoters of $f i b H$, fibL, and $P 25$, respectively, in either M4 or L5D5. Without considering other factors, these results may reflect the similarities and differences of TFs regulating the expression of the three fibroin genes and are worthy of further study. 
Table 2

Potential TFs identified from the PIPs of fibroin genes

\begin{tabular}{|c|c|c|c|c|c|c|c|c|}
\hline \multirow{2}{*}{$\begin{array}{l}\text { Protein ID } \\
\text { P_KWMTBOM000339 }\end{array}$} & \multirow{2}{*}{$\begin{array}{l}\text { Chromosome } \\
\text { Chr1 }\end{array}$} & \multirow{2}{*}{$\begin{array}{l}\text { Family/domain } \\
\text { CSD }\end{array}$} & \multicolumn{3}{|c|}{$\begin{array}{l}\text { Interacting } \\
\text { promoters } \\
\text { in M4 }\end{array}$} & \multicolumn{3}{|c|}{$\begin{array}{l}\text { Interacting } \\
\text { promoters } \\
\text { in L5D5 }\end{array}$} \\
\hline & & & $\mathrm{fibH}$ & & $P 25$ & $\mathrm{fibH}$ & & $P 25$ \\
\hline P_KWMTBOMO00366 & Chr1 & TF_others & $\mathrm{fibH}$ & $f i b L$ & $P 25$ & & $f i b L$ & $P 25$ \\
\hline P_KWMTBOMO00497 & Chr1 & THAP & $\mathrm{fibH}$ & $f i b L$ & $P 25$ & $\mathrm{fibH}$ & & $P 25$ \\
\hline P_KWMTBOM001796 & Chr4 & TF_others & & fibL & & $\mathrm{fibH}$ & fibL & $P 25$ \\
\hline P_KWMTBOM003626 & Chr6 & NCU-G1 & $\mathrm{fibH}$ & fibL & $P 25$ & $\mathrm{fibH}$ & fibL & $P 25$ \\
\hline P_KWMTBOM004742 & Chr8 & ETS & $\mathrm{fibH}$ & & $P 25$ & & & \\
\hline P_KWMTBOM004674 & Chr8 & HMG & & & & & fibL & $P 25$ \\
\hline P_KWMTBOM004757 & Chr8 & MBD & & & & & & $P 25$ \\
\hline P_KWMTBOM004877 & Chr9 & MBD & & & & & & $P 25$ \\
\hline P_KWMTBOM004966 & Chr9 & zf-C2H2 & & & & & $f i b L$ & \\
\hline P_KWMTBOM005228 & Chr9 & $\mathrm{zf}-\mathrm{C} 2 \mathrm{H} 2$ & & & & & & $P 25$ \\
\hline P_KWMTBOM005675 & Chr10 & $\mathrm{zf}-\mathrm{C} 2 \mathrm{H} 2$ & $\mathrm{fibH}$ & $f i b L$ & $P 25$ & & & \\
\hline P_KWMTBOMO05699 & Chr10 & zf-C2H2 & & & & & $f i b L$ & \\
\hline P_KWMTBOM005810 & Chr10 & Nrf1 & & & & $\mathrm{fibH}$ & fibL & $P 25$ \\
\hline P_KWMTBOM007323 & Chr12 & NCU-G1 & & $f i b L$ & $P 25$ & $\mathrm{fibH}$ & $f i b L$ & $P 25$ \\
\hline P_KWMTBOM008022 & Chr13 & HMG & & fibL & $P 25$ & & & \\
\hline P_KWMTBOM008820 & Chr15 & TF_others & $\mathrm{fibH}$ & fibl & $P 25$ & $\mathrm{fibH}$ & $f i b L$ & $P 25$ \\
\hline P_KWMTBOMO09050 & Chr15 & zf-C2H2 & $\mathrm{fibH}$ & fibl & & $\mathrm{fibH}$ & & $P 25$ \\
\hline P_KWMTBOM009808 & Chr16 & NCU-G1 & $\mathrm{fibH}$ & fibL & $P 25$ & $\mathrm{fibH}$ & fibl & $P 25$ \\
\hline P_KWMTBOM010147 & Chr17 & TF_others & & & & & & $P 25$ \\
\hline P_KWMTBOM011061 & Chr18 & TF_others & $\mathrm{fibH}$ & $f i b L$ & $P 25$ & $\mathrm{fibH}$ & $f i b L$ & $P 25$ \\
\hline P_KWMTBOM011731 & Chr19 & HMG & $\mathrm{fibH}$ & $f i b L$ & $P 25$ & & & \\
\hline P_KWMTBOM012659 & Chr21 & NCU-G1 & $\mathrm{fibH}$ & $f i b L$ & $P 25$ & $\mathrm{fibH}$ & & $P 25$ \\
\hline P_KWMTBOM014887 & Chr24 & NCU-G1 & & fibl & $P 25$ & $\mathrm{fibH}$ & $f i b L$ & $P 25$ \\
\hline P_KWMTBOM015035 & Chr25 & THAP & & & & & & $P 25$ \\
\hline
\end{tabular}




\begin{tabular}{|c|c|c|c|c|c|c|c|c|}
\hline \multirow{2}{*}{$\begin{array}{l}\text { Protein ID } \\
\text { P_KWMTBOM015757 }\end{array}$} & \multirow{2}{*}{$\begin{array}{l}\text { Chromosome } \\
\text { Chr26 }\end{array}$} & \multirow{2}{*}{$\begin{array}{l}\text { Family/domain } \\
\text { Homeobox }\end{array}$} & \multicolumn{3}{|c|}{$\begin{array}{l}\text { Interacting } \\
\text { promoters } \\
\text { in M4 }\end{array}$} & \multicolumn{3}{|c|}{$\begin{array}{l}\text { Interacting } \\
\text { promoters } \\
\text { in L5D5 }\end{array}$} \\
\hline & & & & & & $\mathrm{fibH}$ & & $P 25$ \\
\hline P_KWMTBOM015758 & Chr26 & Homeobox & $\mathrm{fibH}$ & fibL & $P 25$ & $\mathrm{fibH}$ & $f i b L$ & $P 25$ \\
\hline P_KWMTBOM015781 & Chr26 & THAP & & $f i b L$ & & $\mathrm{fibH}$ & $f i b L$ & $P 25$ \\
\hline P_KWMTBOM015928 & Chr27 & bHLH & & fibL & & & & $P 25$ \\
\hline P_KWMTBOM016040 & Chr27 & TF_others & & fibL & $P 25$ & & & $P 25$ \\
\hline P_KWMTBOM016524 & Scaf007 & TF_others & $\mathrm{fibH}$ & $f i b L$ & $P 25$ & & fibl & \\
\hline
\end{tabular}

Combinatorial TF interactions are critical for gene regulation and are important determinants of different cellular functions. To determine possible interactions among the $31 \mathrm{TFs}$, we constructed corresponding PPI network models using the STRING database. As shown in Supplementary Fig. S4, two interaction networks were identified, which consisted of 28 nodes and 16 edges. One module was composed of 2 TFs annotated as prohibitin, which is a highly conserved protein that can inhibit the proliferation and apoptosis of tumor cells by regulating gene transcription and maintaining the stability of mitochondrial proteins. The other module was composed of 9 TFs. Interestingly, two ribosomal proteins (P_KWMTBOM004742 and P_KWMTBOM005675) and one translation elongation factor (P_KWMTBOM011731) that form a subnetwork and interact with each other were found only in the M4 PSG, while adenylate kinase 9 (P_KWMTBOM005810) was found only in the L5D5 PSG. In addition, another subnetwork was composed of 5 TFs (P_KWMTBOM007323, P_KWMTBOM003626, P_KWMTBOM014887, P_KWMTBOM009808, and P_KWMTBOM012659) that interact with each other and are all molecular chaperones with similar functions assisting the folding of proteins upon ATP hydrolysis. Taken together, these results suggest that these TFs are crucial for the correct transcription and folding of silk fibroin proteins and deserve further study.

\section{Discussion}

As a fully domesticated insect, the silkworm, Bombyx mori, has attracted much attention from researchers, not only because it can produce silk for human uses but also because of the fascinating mechanisms underlying the precise, concerted regulation of silk protein synthesis at the transcriptional level during larval development. Previous studies have provided some regulatory evidence related to silk protein synthesis, particularly regarding the discovery of several factors, such as TFs, that directly regulate silk protein gene expression. However, it is still difficult to reveal the detailed mechanisms of silk protein synthesis on the basis of the reported regulatory factors. 
In an attempt to discover the factors regulating silk protein genes, we mainly focused on the proteins directly binding to promoters of the fibroin protein genes $\mathrm{fibH}$, fibL, and $P 25$, which encode one of the two component proteins of silk fibers. Candidate factors interacting with the promoters of the three fibroin genes, or PIPs, were obtained from M4 and L5D 5 PSGs by DNA pull-down assays and identified using the HPLC-MS method. Our results revealed hundreds of PIPs for each fibroin gene in either the M4 or L5D5 PSG, which preliminarily demonstrates the complexity of the regulation of fibroin gene expression. It is of course possible that some PIPs may have been overlooked in our experiment, partially due to the limitations of the applied technique itself and the use of only one biotin-labeled DNA probe for each fibroin gene. Nevertheless, the obtained data are of great importance for better understanding the regulatory factors that interact directly with fibroin protein genes.

Whether there is any difference between the proteins binding to fibroin gene promoters during larval molting and feeding stages is an interesting question. In this work, we selected two representative stages showing significant differences in fibroin protein synthesis in the PSG. M4 is one of the stages during which fibroin protein synthesis is inhibited, while L5D5 is a critical period in which abundant fibroin proteins are synthesized. As a result, 198, 292, and 247 proteins obtained from the M4 PSG and 330, 305, and 460 proteins obtained from the L5D5 PSG were identified as PIPs of fibH, fibL, and P25, respectively. From these data and subsequent KEGG pathway annotation, we found that many of these PIPs had similar functional annotation classifications and were annotated to several different KEGG systems, particularly metabolism, genetic information processing, and human diseases. Interestingly, the number of PIPs in the L5D5 PSG was greater than that observed in the M4 PSG, suggesting that more regulatory proteins may be needed to ensure efficient silk protein synthesis. In addition, there were some obvious differences in the PIPs identified between different fibroin genes, which implies that the cooperative regulation mechanisms of the three fibroin genes may be far more complex than we predicted. Further study will improve our understanding of the regulatory mechanism of fibroin protein synthesis.

The TFs among the fibroin gene PIPs were key factors upon which we focused because many TFs play vital roles as "master regulators" and "selector genes" of downstream target genes ${ }^{20}$. In B. mori, TFs are particularly critical for the spatiotemporal regulation of silk protein synthesis; however, only a few TFs have been identified and confirmed by in vivo or in vitro experiments. In this study, we identified 31 TFs from the M4 and/or L5D5 PSG, which contained conserved domains such as zf-C2H2, bHLH, MYB and THAP domains. To our knowledge, there is little information on their roles in regulating fibroin genes. Therefore, it would be interesting to investigate the function of these TFs in the PSG. Surprisingly, no TFs previously proven to bind to the promoter regions of fibroin genes, including FMBP-1, SGF-2, Bmdimm, POU-M2, and BmFTZ-F1 ${ }^{13-19}$, were found among the PIPs of fibroin genes. We speculate that in addition to the limitations of the experimental techniques mentioned above, there is another possibility that is worthy of discussion. TFs do not seem to like to act alone. Most eukaryotic TFs are thought to act by recruiting cofactors and forming protein complexes with them ${ }^{21}$. However, some studies have suggested that the interactions between a TF and its cofactors are weak/transient ${ }^{12,22}$. Therefore, it is possible that some cofactors, rather than TFs, that play master regulatory roles in the PSG of $B$. mori, may have been 
captured in the present study. In addition, only two periods of PSG development were examined in this study, and it is also possible that some TFs functioning outside these two periods cannot be captured. In short, the identification and functional analysis of TFs is a complex and arduous task. The TFs identified in our study are worthy of further investigation, which may provide novel insights into the regulatory mechanisms of fibroin genes.

In summary, this study describes the large-scale identification of regulatory factors interacting directly with the promoter regions of fibroin genes for the first time. Our results demonstrate that a large number of proteins are involved in the regulation of fibroin genes by directly interacting with their promoters. This research provides an important foundation for further elucidating the biological functions and regulatory roles of these DNA-interacting proteins and elaborating the intricate regulatory network of fibroin protein synthesis.

\section{Materials And Methods}

Animals and sample preparation. The silkworm strain Nistari was maintained in our laboratory. The hatched larvae were normally reared on fresh mulberry leaves at $25-26^{\circ} \mathrm{C}$. The Nistari PSGs used in the DNA pull-down assay were dissected from the M4 and L5D5 larvae. All PSG samples were washed in phosphate-buffered saline (PBS, $\mathrm{pH} 7.4$ ) and stored at $-80^{\circ} \mathrm{C}$ until required.

Cell culture and luciferase reporter assay. Cultured B. mori embryonic cells $(\mathrm{BmE})$ maintained at $27^{\circ} \mathrm{C}$ in Grace's insect medium containing $10 \%$ fetal bovine serum (HyClone, China) were used to examine the promoter activities of fibroin genes. In brief, the promoter sequences of the fibroin genes $\mathrm{fibH}$ (AF226688.1), fibL (AAA27840.1) and P25 (X04226.1), with lengths of $2096 \mathrm{bp}, 1060 \mathrm{bp}$ and $1227 \mathrm{bp}$, respectively, were commercially synthesized (GenScript, China) and inserted into the empty pGL4.11[Luc2P] vector (Promega, USA). The abbreviations for the final constructs were fibH-Luc2P, fibLLuc2P, and P25-Luc2P, respectively. Then, a mixture of $3.5 \mu \mathrm{L}$ of transfection reagents (Roche, USA) with $1.5 \mu \mathrm{g}$ of the fibH-Luc2P, fibL-Luc2P, or P25-Luc2P plasmid DNA was transfected into BmE cells. After $36 \mathrm{~h}$ of culture at $27^{\circ} \mathrm{C}$, the cells were harvested, and luciferase activity was determined using a Dual Luciferase Reporter Gene Assay Kit (Yeasen, China). pGL4.11[Luc2P] was used as a negative control. The plasmids p57S[hrA4-Gal4] (A4G4) and pGL4.11[UAS-Luc2P] (UAS-Luc2P), which we constructed based on a previously modified Gal4/UAS binary system ${ }^{23,24}$, were used as positive controls.

DNA pull-down assay. First, the plasmids fibH-Luc2P, fibL-Luc2P, and P25-Luc2P were used as templates, and the promoter regions of $\mathrm{fibH}$, fibL and $P 25$ were amplified by PCR using 5 '-biotin-labeled forward primers (Supplementary Table S5). Each of the 5'-biotinylated DNA sequences was immobilized on streptavidin beads following the manufacturer's protocol (Dynabeads ${ }^{\mathrm{TM}}$ MyOne $^{\mathrm{TM}}$ Streptavidin C1, Invitrogen, USA). Then, PSG proteins in the nuclear fraction were incubated with 5'-biotinylated DNA beads on a rotating shaker at $4^{\circ} \mathrm{C}$ overnight. Following this incubation, the supernatant was removed, and the beads were washed three times with RIP wash buffer (Fitgene, China). After the last wash, the pull-down mixture was resuspended in elution buffer on a rotating shaker at room temperature for 15 min to break 
the bonds between streptavidin and biotin. Finally, the proteins eluted from the beads were subjected to polyacrylamide gel electrophoresis (PAGE) followed by silver nitrate staining and MS analysis. The proteins eluted from the beads with the unbiotinylated DNA probe and GFP probe were used as controls.

HPLC-MS analysis. Peptides were dissolved in $0.1 \%$ formic acid (FA, Sigma, USA) and $2 \%$ acetonitrile (ACN, Fisher, Germany) and directly loaded onto a reversed-phase analytical column $(75 \mu \mathrm{m}$ i.d. $\times 150$ mm, packed with Acclaim PepMap RSLC C18, $2 \mu \mathrm{m}, 100 \AA$, nanoViper). The applied gradient was as follows: increase from $5-50 \%$ solvent $B(0.1 \%$ FA in $80 \%$ ACN) over 40 min, followed by an increase to $90 \%$ B over $5 \mathrm{~min}$, and then holding at $90 \%$ B for $5 \mathrm{~min}$, all at a constant flow rate of $300 \mathrm{~nL} / \mathrm{min}$.

MS analysis was performed on a Q Exactive hybrid quadrupole-Orbitrap mass spectrometer (Thermo Fisher Scientific). The peptides were subjected to a nanospray ionization (NSI) source, followed by tandem mass spectrometry (MS/MS) in a Q Exactive ${ }^{\text {TM }}$ system (Thermo) coupled online to an ultraperformance liquid chromatography (UPLC) system. Intact peptides were detected in the Orbitrap at a resolution of 70,000. Peptides were selected for MS/MS using a normalized collision energy (NCE) setting of 27; ion fragments were detected in the Orbitrap at a resolution of 17,500. A data-dependent procedure that alternated between one MS scan and 20 subsequent MS/MS scans was applied for the top 20 precursor ions above a threshold ion count of 1E4 in the MS survey scan with $30.0 \mathrm{~s}$ dynamic exclusion. The applied electrospray voltage was $2.0 \mathrm{kV}$. Automatic gain control (AGC) was used to prevent the overfilling of the ion trap; $1 \mathrm{E} 5$ ions were accumulated for the generation of MS/MS spectra. For MS scans, the $\mathrm{m} / \mathrm{z}$ scan range was 350 to $1800 \mathrm{~m} / \mathrm{z}$. The fixed first mass was set as $100 \mathrm{~m} / \mathrm{z}$.

Data analysis. Peptide and protein identification were performed with ProteinPilot ${ }^{\text {TM }}$ Software 4.5 (version 1656, AB SCIEX, USA) by searching SilkBase (http://silkbase.ab.a.u-tokyo.ac.jp). The functional annotation of the PIPs was conducted with KEGG BlastKoala ${ }^{25}$ by selecting "Taxonomy group: Eukaryotes", setting the KEGG database search parameters to "family_eukaryotes + genus_prokaryotes" and setting other parameters to the default values. The proteins identified by the pull-down assays were aligned in the AnimalTFDB3.0 website (http://bioinfo.life.hust.edu.cn/AnimalTFDB2/index.shtml) using the following filtration parameters to predict the candidate TFs: identity $(\%) \geq 30$ and coverage $(\%) \geq 50$. The STRING database and web tool ${ }^{26}$ were used to predict the protein-protein interaction networks.

\section{Declarations}

\section{Acknowledgments}

This work was supported by the National Natural Science Foundation of China (Grant No. 31872291) and a grant from the Chongqing Science and Technology Bureau (No. cstc2020yszx-jcyjX0003). The English language of the manuscript was polished by American Journal Experts.

\section{Author contributions}


H.F.X., Y.M., conceptualization and methodology; Y.M., Q.L., Y.O., Y.Y.T., W.H.Z., experiments and analysis. H.M.W., J.H., data analysis. H.F.X., Y.M., manuscript writing. All authors read and approved the manuscript.

\section{Competing interests}

The authors declare no competing interests.

\section{References}

1. Bulger, M. Groudine, Enhancers: The abundance and function of regulatory sequences beyond promoters. Dev Bio/ 339, 250-257 (2010).

2. Srikanth, S. Ramachandran, S. S. Mohan, Construction of the gene regulatory network identifies MYC as a transcriptional regulator of SWI/SNF complex. Sci Rep-Uk 10, 158 (2020).

3. Ptashne, A. Gann, Genes and Signals. (Cold Spring Harbor Laboratory Press, New York, 2002),

4. H. Tran et al., Identification of DNA-binding proteins that interact with the 5 '-flanking region of the human D-amino acid oxidase gene by pull-down assay coupled with two-dimensional gel electrophoresis and mass spectrometry. J Pharmaceut Biomed 116, 94-100 (2015).

5. F. Xu, D. A. O'Brochta, Advanced technologies for genetically manipulating the silkworm Bombyx mori, a model Lepidopteran insect. P Roy Soc B-Biol Sci 282, (1810):20150487 (2015).

6. Asakura et al., Some observations on the structure and function of the spinning apparatus in the silkworm Bombyx mori. Biomacromolecules 8, 175-181 (2007).

7. Valluzzi, S. J. He, S. P. Gido, D. Kaplan, Bombyx mori silk fibroin liquid crystallinity and crystallization at aqueous fibroin-organic solvent interfaces. Int J Biol Macromol 24, 227-236 (1999).

8. Inoue et al., Silk fibroin of Bombyx mori is secreted, assembling a high molecular mass elementary unit consisting of H-chain, L-chain, and P25, with a $6: 6: 1$ molar ratio. J Biol Chem 275, 4051740528 (2000).

9. Maekawa, Y. Suzuki, Repeated turn-off and turn-on of fibroin gene-transcription during silk gland development of Bombyx-mori. Dev Biol 78, 394-406 (1980).

10. P Couble, JJ Michaille, A Garel, ML Couble, JC Prudhomme. Developmental switches of sericin mRNA splicing in individual cells of Bombyx mori silk gland. Dev Bio/ 124, 431-40 (1987).

11. T Obara, Y Suzuki. Temporal and spatial control of silk gene transcription analyzed by nuclear run-on assays. Dev Biol 127, 384-91 (1988).

12. A. Lambert et al., The Human Transcription Factors. Cell 172, 650-665 (2018).

13. Takiya, T. Ishikawa, K. Ohtsuka, Y. Nishita, Y. Suzuki, Fibroin-modulator-binding protein-1 (FMBP-1) contains a novel DNA-binding domain, repeats of the score and three amino acid peptide (STP), conserved from Caenorhabditis elegans to humans. Nucleic Acids Res 33, 786-795 (2005).

14. Tsutsumi et al., In vivo fluorescence correlation spectroscopy analyses of FMBP-1, a silkworm transcription factor. Febs Open Bio 6, 106-125 (2016). 
15. Ohno et al., Silk gland factor-2, involved in fibroin gene transcription, consists of LIM homeodomain, LIM-interacting, and single-stranded DNA-binding proteins. J Biol Chem 288, 31581-31591 (2013).

16. Kimoto, T. Tsubota, K. Uchino, H. Sezutsu, S. Takiya, LIM-homeodomain transcription factor Awh is a key component activating all three fibroin genes, $f i b H, f i b L$ and $f h x$, in the silk gland of the silkworm, Bombyx mori. Insect Biochem Molec 56, 29-35 (2015).

17. M. Zhao et al., A juvenile hormone transcription factor Bmdimm-fibroin $\mathrm{H}$ chain pathway is involved in the synthesis of silk protein in silkworm, Bombyx mori. J Biol Chem 290, 972-986 (2015).

18. N. Liu et al., Biochemical characterization and functional analysis of the POU transcription factor POU-M2 of Bombyx mori. Int J Biol Macromol 86, 701-708 (2016).

19. Y. Zhou et al., Nuclear hormone receptor BmFTZ-F1 is involved in regulating the fibroin heavy chain gene in the silkworm, Bombyx mori. Bba-Gen Subjects 1860, 2529-2536 (2016).

20. I. Lee, R. A. Young, Transcriptional regulation and its misregulation in disease. Cel/ 152, 1237-1251 (2013).

21. Reiter, S. Wienerroither, A. Stark, Combinatorial function of transcription factors and cofactors. Curr Opin Genet Dev 43, 73-81 (2017).

22. Marcon et al., Human-chromatin-related protein interactions identify a demethylase complex required for chromosome segregation. Cell Rep 8, 297-310 (2014).

23. H. Zeng et al., BmYki is transcribed into four functional splicing isoforms in the silk glands of the silkworm Bombyx mori. Gene 646, 39-46 (2018).

24. T. Tan et al., The intronic promoter of Actin 4 mediates high-level transgene expression mainly in the wing and epidermis of silkworms. Transgenic Res 29, 243-251 (2020).

25. Kanehisa et al., BlastKOALA and GhostKOALA: KEGG tools for functional characterization of genome and metagenome sequences. J Mol Biol 428, 726-731 (2016).

26. J. Jensen et al., STRING 8-a global view on proteins and their functional interactions in 630 organisms. Nucleic Acids Res 37, D412-D416 (2009).

\section{Figures}


a

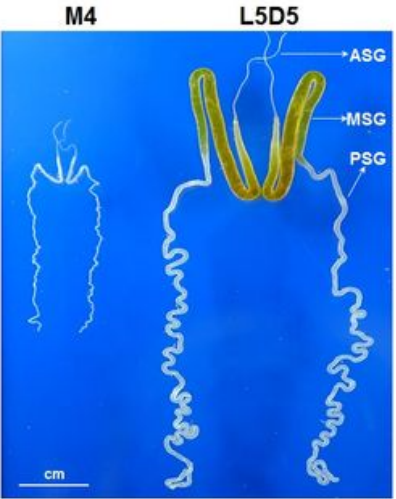

b
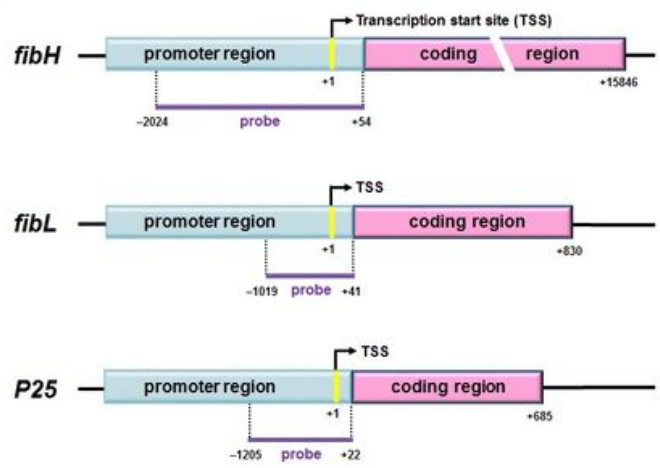

C

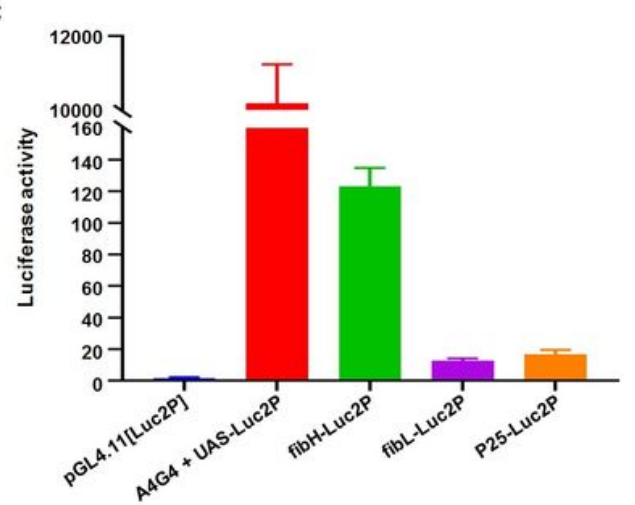

d

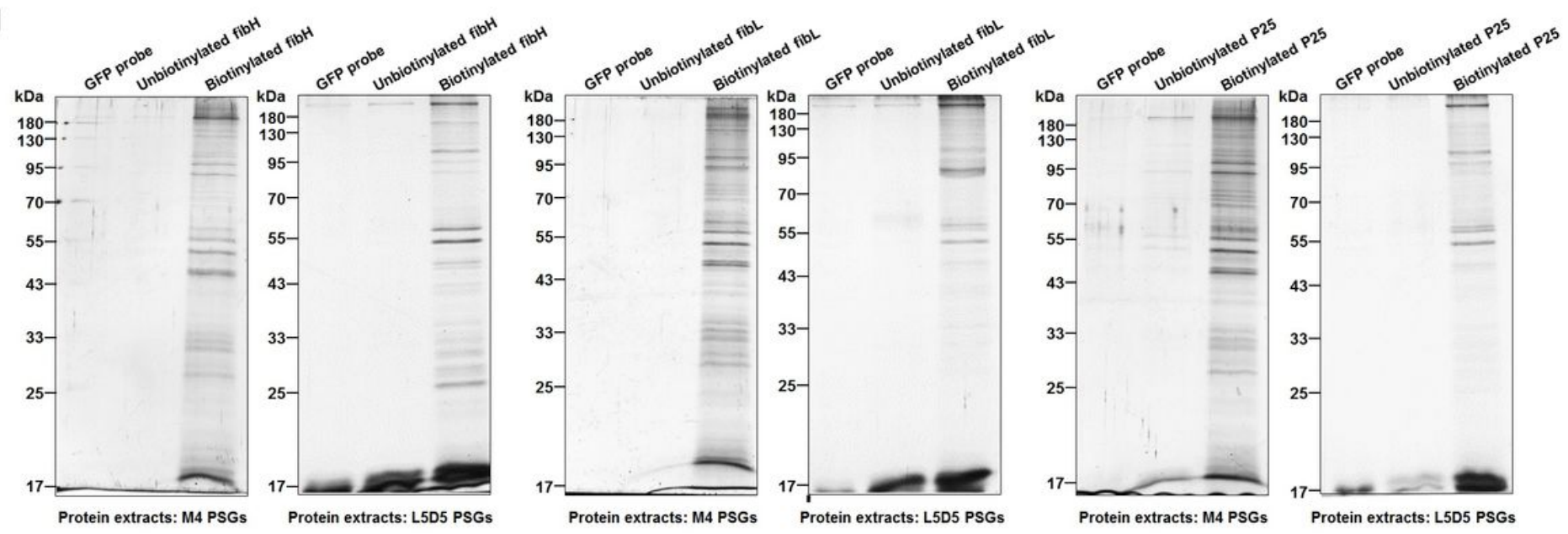

Figure 1

Probe design and DNA pull-down assay. (a) SGs dissected from silkworm larvae developed to M4 and L5D5. (b) Diagram of the fibroin genes fibH, fibL, and P25 and the locations of DNA probes. (c) Promoter activities of the fibH, fibL, and P25 genes measured by luciferase reporter assays. (d) Potential PIPs in PSG nuclear protein extracts were pulled down using the probes. After SDS-PAGE and silver staining, the bound proteins were significantly enriched only in those groups in which 5'-biotinylated probes were used. 
a

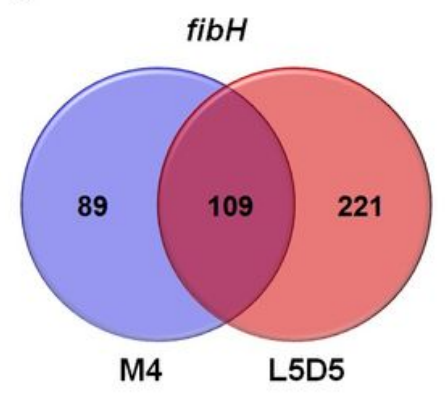

fibL

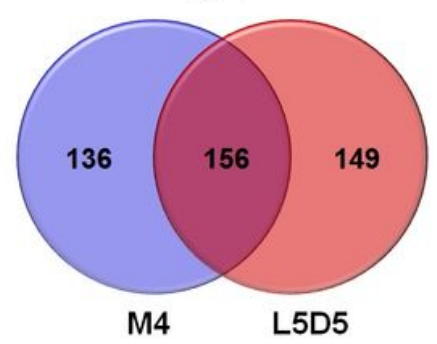

P25

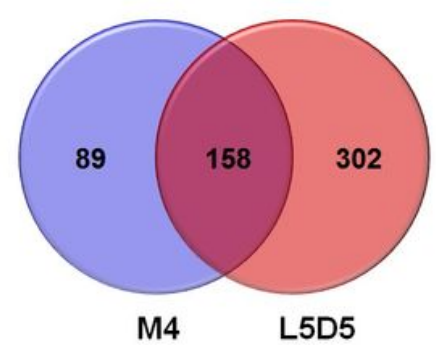

b

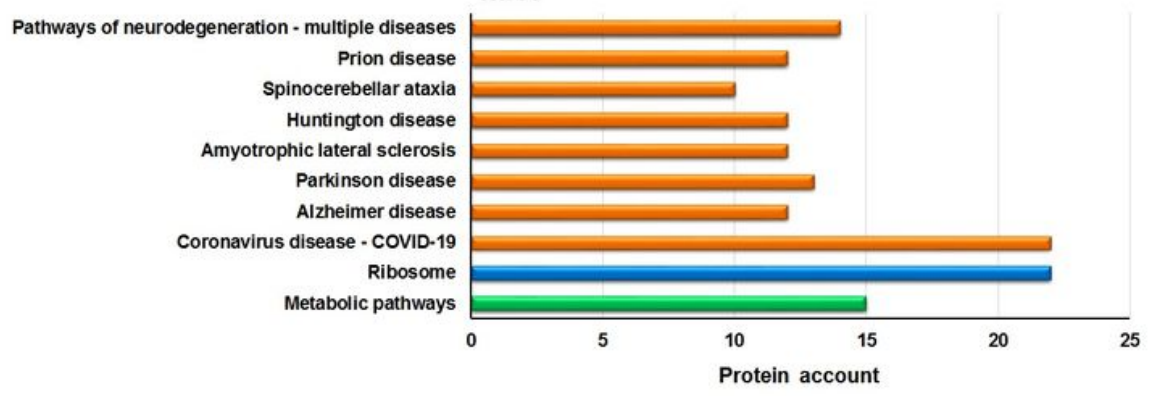

$f i b L$

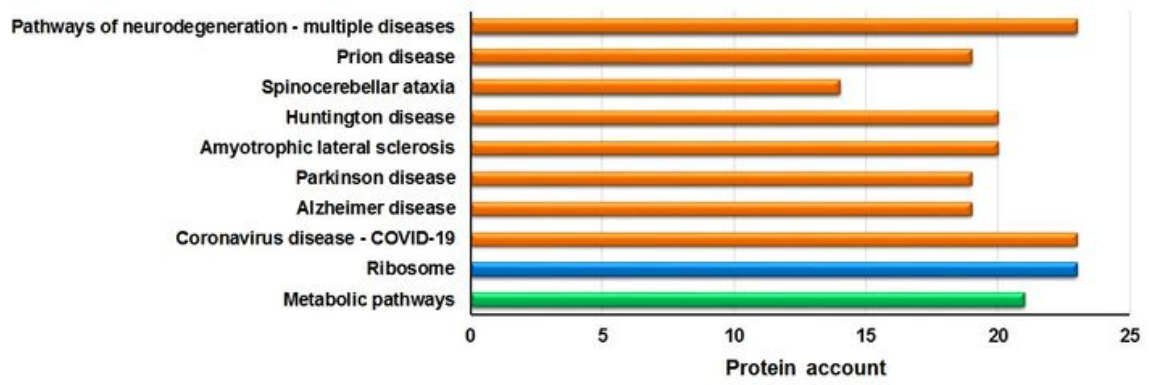

P25

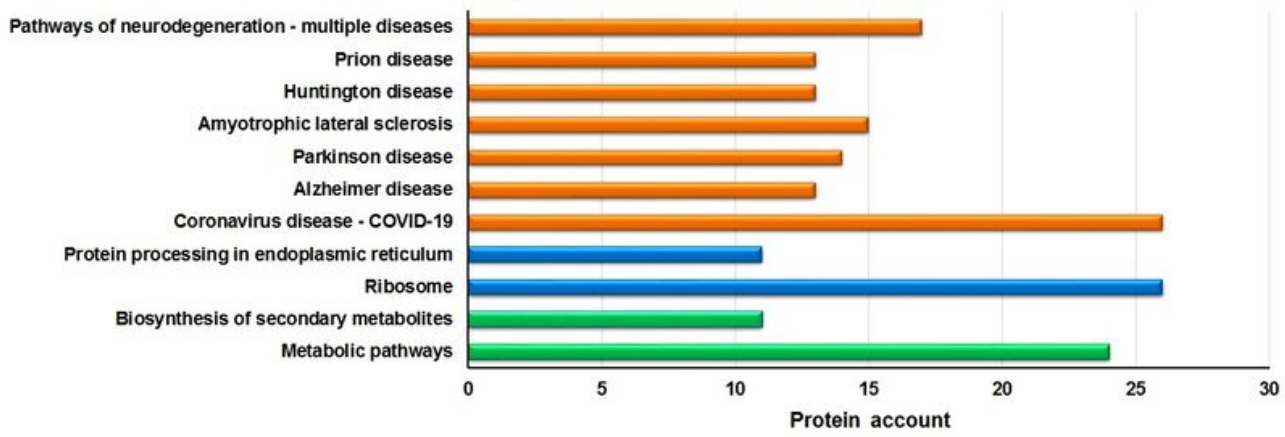

Figure 2

Overlap between the PIPs of each fibroin gene in M4 and L5D5. (a) Venn diagrams showing the numbers of common and unique PIPs of the fibH, fibL, and P25 genes. (b) KEGG pathway enrichment of common PIPs. Top 10 KEGG pathways are displayed. 


\section{a}

afibH $\square$ fibL $=$ P25

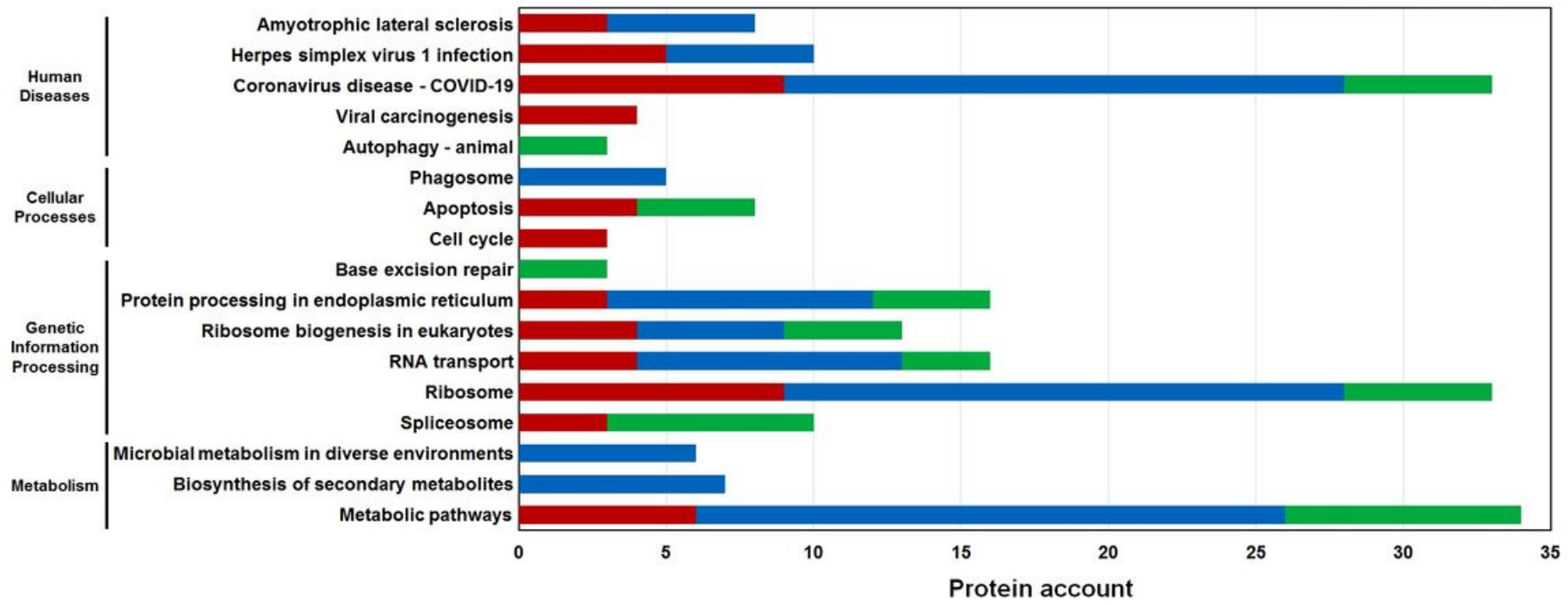

b

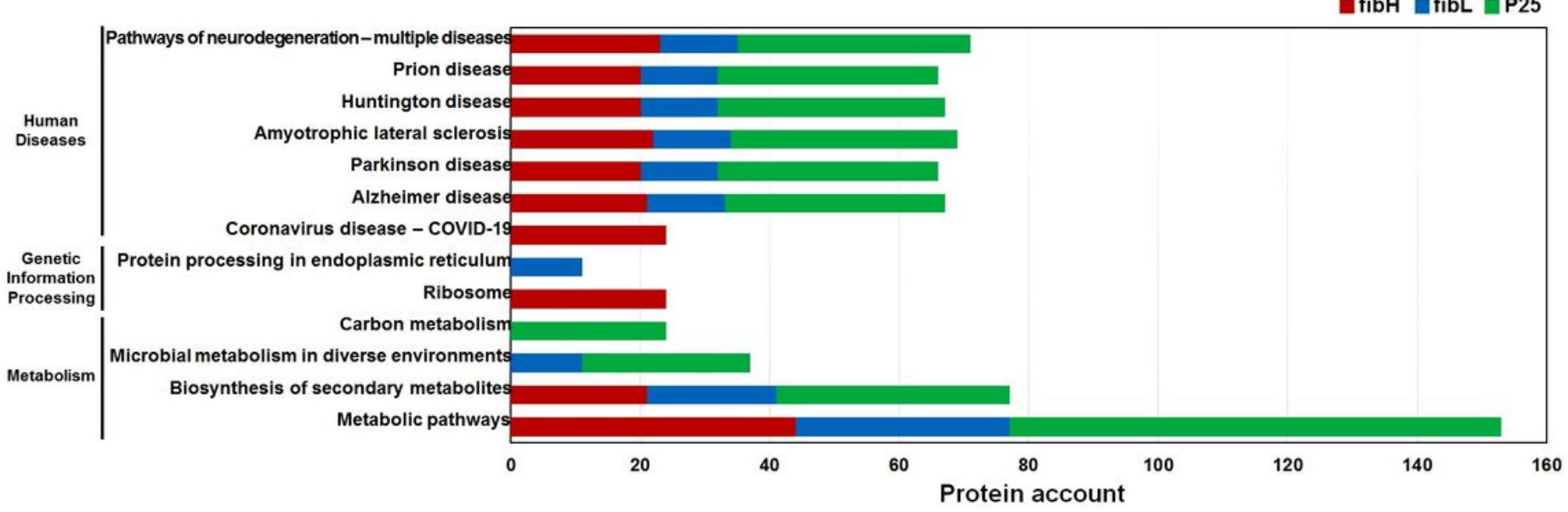

\section{Figure 3}

Top 10 KEGG pathways enriched in unique PIPs of each fibroin gene in M4 (a) and L5D5 (b). Red, blue, and green represent the PIPs of fibH, fibL, and P25, respectively. 
a

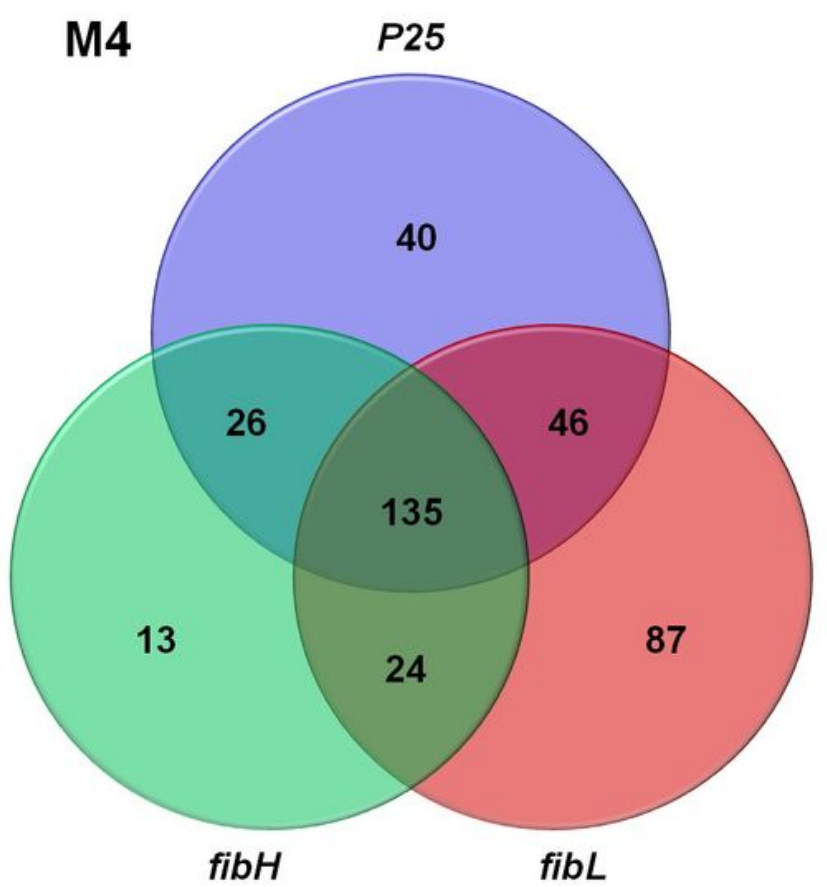

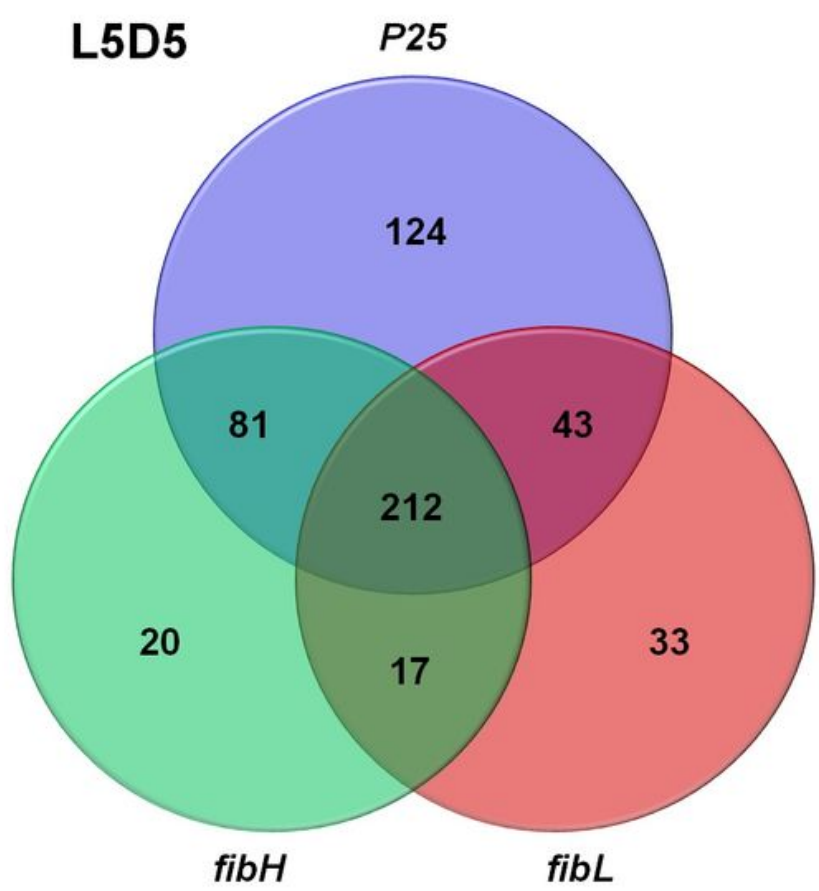

b

$\square$ M4 $\quad$ L5D5 $=$ M4 + L5D5

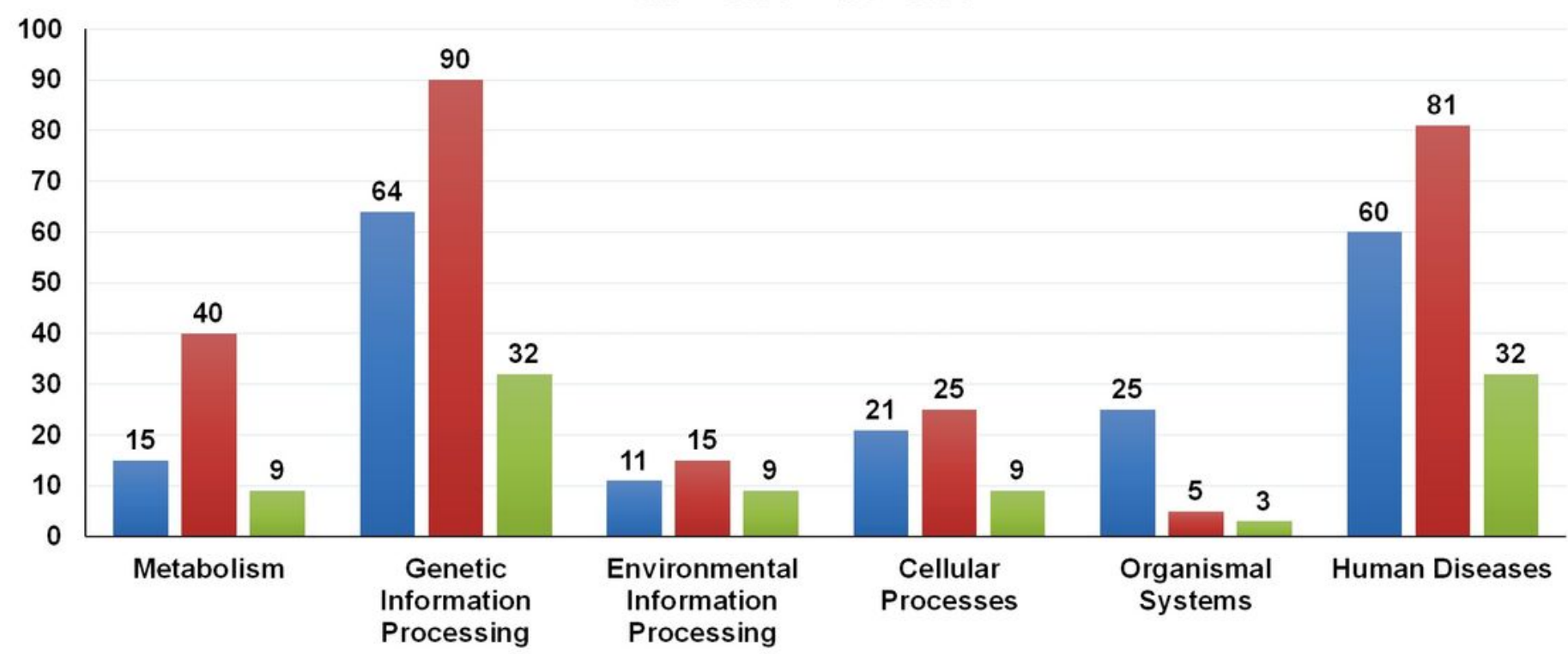

Figure 4

Common PIPs shared by the three fibroin genes. (a) Venn diagrams showing the numbers of PIPs interacting with the promoters of fibH, fibL, and P25 in either the M4 or L5D5 PSG. (b) Distribution of common PIPs in KEGG systems. The numbers on the top of each column are the PIP counts. The green columns represent the PIPs found in both M4 and L5D5 PSGs. 
a
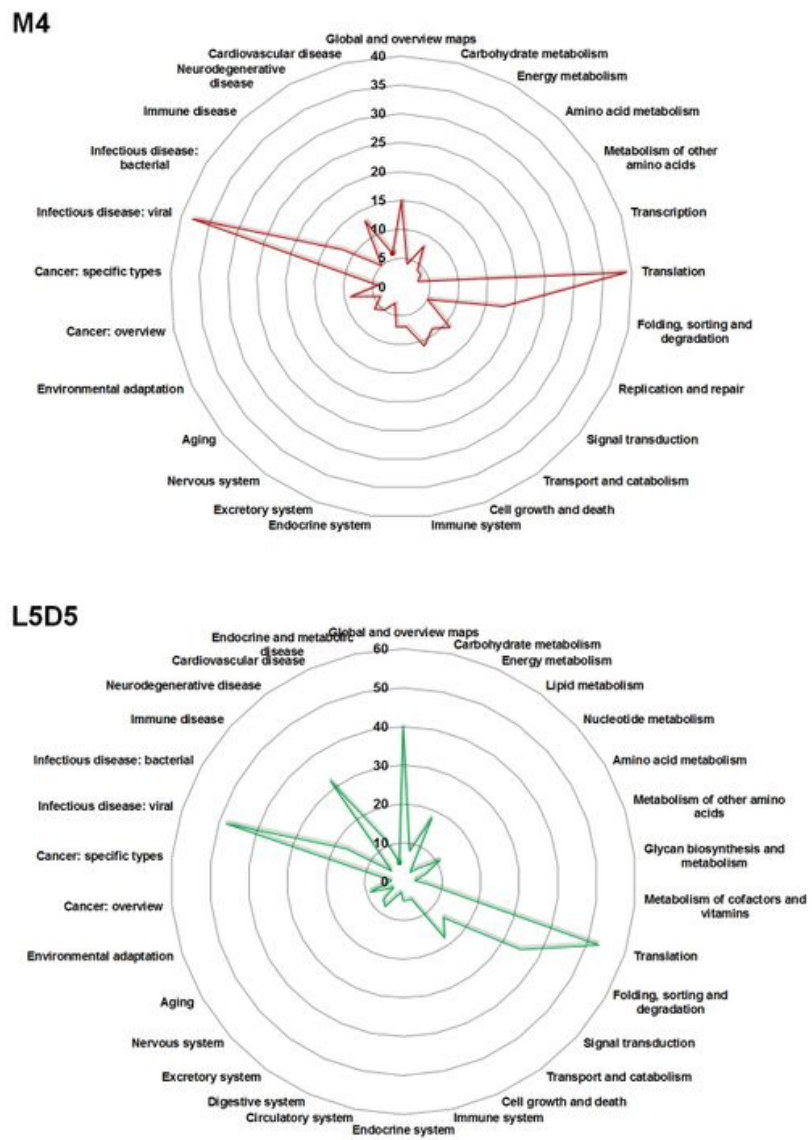

b

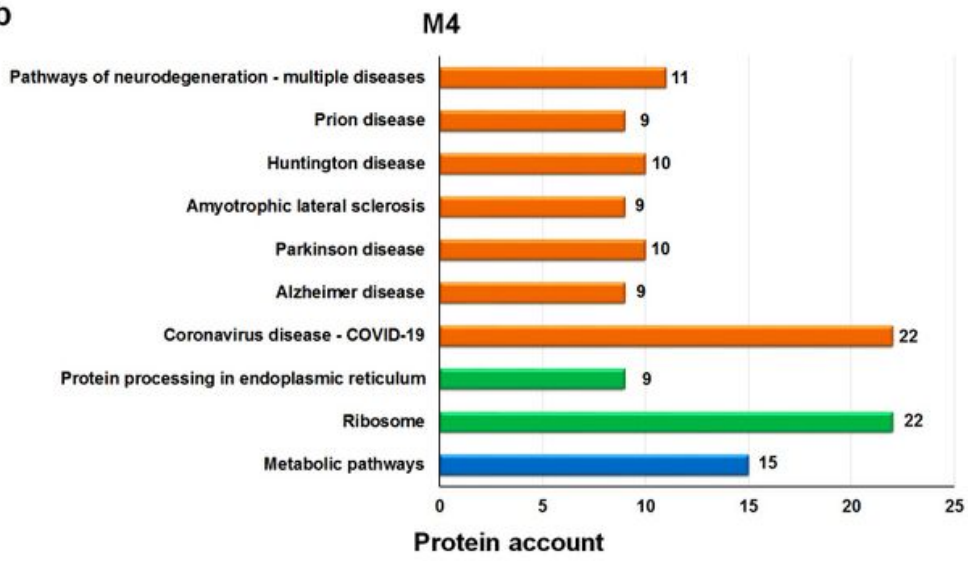

\section{L5D5}

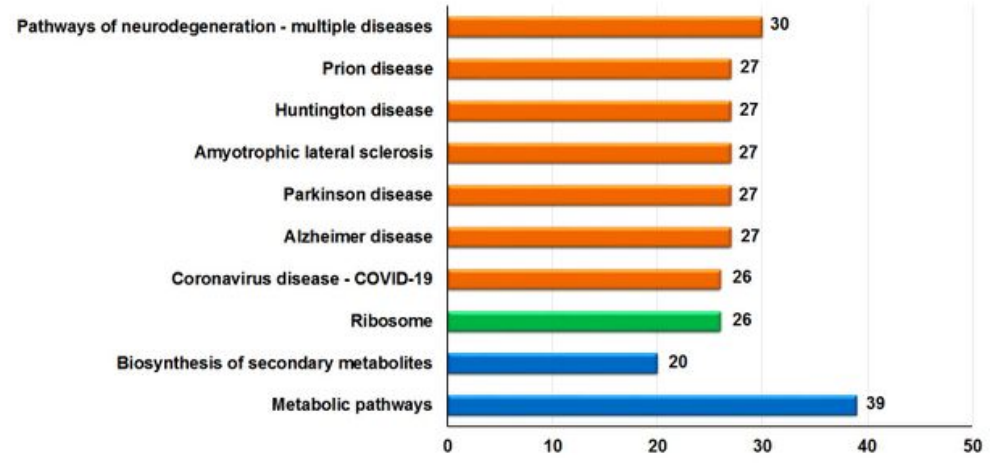

Protein account

\section{Figure 5}

Functional characterization of common PIPs shared by the three fibroin genes. (a) KO pathways. (b) KO Brite Top 10. 

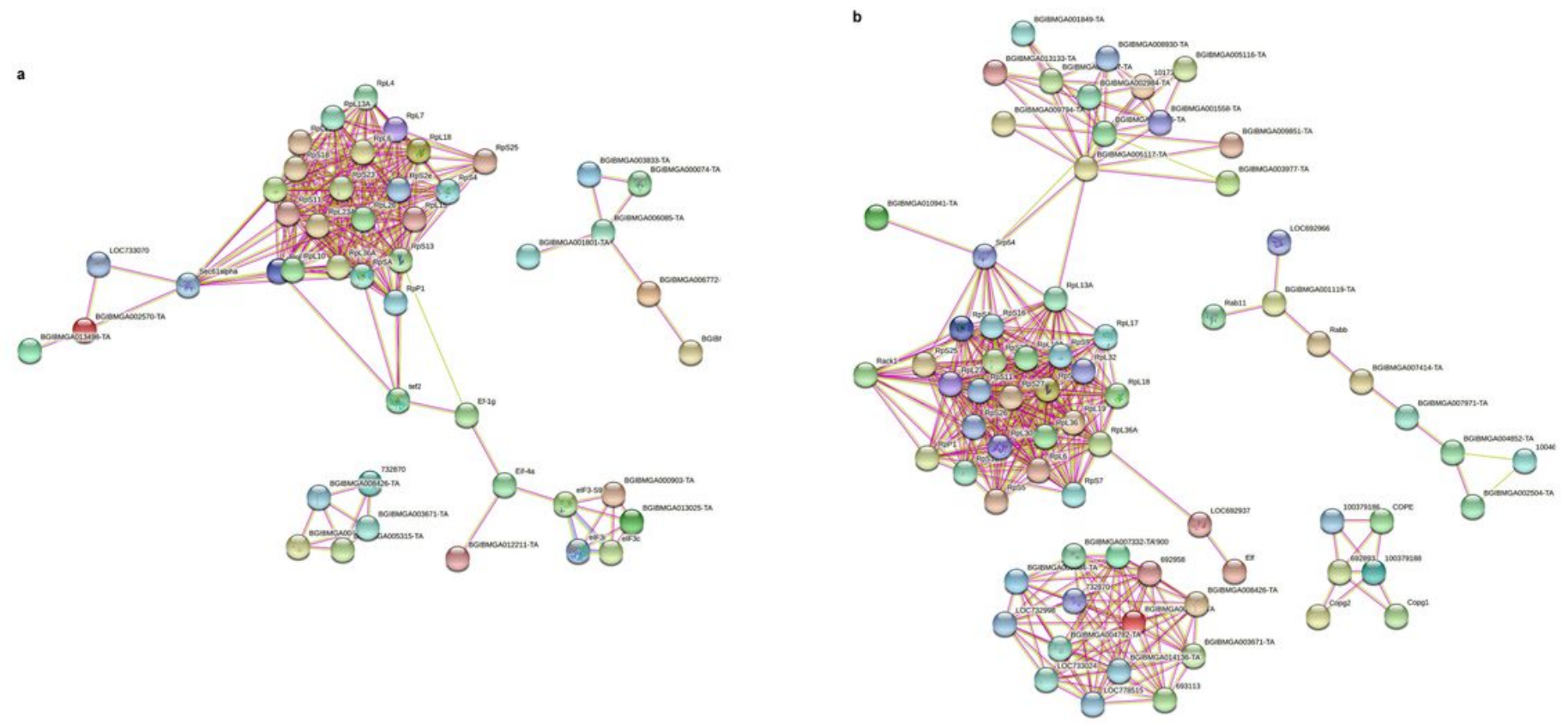

\section{Figure 6}

Protein-protein interaction networks among the common PIPs shared by the three fibroin genes. (a) The top three significant modules selected from the PPI network generated based on common PIPs from M4. (b) The top four significant modules selected from the PPI network generated based on common PIPs from L5D5. Pink or pale green lines represent interaction relationships between the nodes generated based on the available evidence in the STRING database. Minimum required interaction score: highest confidence (0.900); PPI enrichment p-value: <1.0e-16.

\section{Supplementary Files}

This is a list of supplementary files associated with this preprint. Click to download.

- Table1.docx

- Table2.docx

- Supplementaryinformation.pdf

- SupplementaryTableS1.xlsx

- SupplementaryTableS2.xlsx

- SupplementaryTableS3.xlsx

- SupplementaryTableS4.xlsx

- SupplementaryTableS1.xlsx

- SupplementaryTableS2.xlsx 
- SupplementaryTableS3.xIsx

- SupplementaryTableS4.xIsx

- Supplementaryinformation.pdf 